\begin{tabular}{|ll}
$\begin{array}{l}\text { Questions } \\
\text { vives }\end{array}$ & $\begin{array}{l}\text { Questions Vives } \\
\text { Recherches en éducation }\end{array}$ \\
& $\begin{array}{l}\mathbf{N}^{\circ} \mathbf{2 6} \mid \mathbf{2 0 1 6} \\
\text { Questions à la didactique de l'histoire }\end{array}$ \\
\hline
\end{tabular}

\title{
Questions à la didactique de l'histoire
}

\section{Christian Roux}

\section{(2) OpenEdition \\ Journals}

\section{Édition électronique}

URL : http://journals.openedition.org/questionsvives/2034

DOI : 10.4000/questionsvives.2034

ISSN : 1775-433X

\section{Éditeur}

Université Aix-Marseille (AMU)

\section{Édition imprimée}

Date de publication : 15 décembre 2016

ISBN : 978-2-912643-50-6

ISSN : 1635-4079

\section{Référence électronique}

Christian Roux, «Questions à la didactique de I'histoire », Questions Vives [En ligne], N²6 | 2016, mis en ligne le 27 décembre 2017, consulté le 10 décembre 2020. URL : http://journals.openedition.org/ questionsvives/2034; DOI : https://doi.org/10.4000/questionsvives.2034

Ce document a été généré automatiquement le 10 décembre 2020.

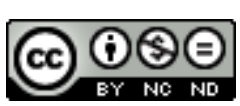

Questions Vives est mis à disposition selon les termes de la licence Creative Commons Attribution Pas d'Utilisation Commerciale - Pas de Modification 4.0 International. 


\title{
Questions à la didactique de l'histoire
}

\author{
Christian Roux
}

1 L'histoire scolaire est le seul usage social de l'histoire en présence de laquelle seront obligatoirement mis tous les futurs citoyens au cours de leurs formations scolaires (Heimberg, 2007). Seule représentation du passé dans la classe, cette histoire scolaire n'a pas, a priori, vocation à être critiquée (Audigier, 1988). Enseignement du professeur ou reconstruction de l'élève à partir des documents fournis, l'histoire ne peut être contredite par un élève qui se trouve, dans le système didactique, toujours en position de non-connaissant des éléments en cours d'apprentissage. Cette dimension semble effacer la distance existant naturellement entre une représentation et les événements tels qu'ils se sont produits dans le passé inatteignable (Ginzburg, 2001). Comme toutes les représentations historiques, l'histoire scolaire est une création qui porte en elle les contraintes de l'époque, de la société et du lieu qui l'ont vu naître, ce que Marc Ferro nomme le foyer, ici l'école, et plus largement l'État (1985). La didactique de l'histoire, comme science étudiant la diffusion des connaissances historiques s'intéresse à ce phénomène. Fruit de la transposition didactique (Chevallard, 1991), l'histoire scolaire subit des contraintes lors de sa composition qui font d'elle une question vive. En ellemême la transposition didactique ne fait pas consensus dans la didactique de l'histoire, où elle se heurte à la « discipline scolaire » issue des travaux d'André Chervel qui voit dans l'histoire scolaire une vulgate consensuelle éloignée du savoir scientifique (Cariou, 2012).

$2 \mathrm{Au}$ cours de la transposition didactique, l'objet de savoir qui est la représentation historique va subir un certain nombre de transformations qui vont faire que cette histoire scolaire n'est plus celle qui a été composée dans la sphère scientifique. L'histoire, comme écriture scientifique, est toujours en renouvellement et s'offre à la critique des historiens. Virtuellement sans limites, elle peut observer toutes les facettes du passé (Moniot, 1993). Afin de rentrer dans le cadre de l'histoire scolaire contrainte dans un temps et un volume imposé, un choix doit être fait, et comme tous les choix celui-ci est critiquable (Audigier, 1988). Afin d'être enseigné, l'objet de savoir doit être 
sélectionné par la noosphère, communauté implicite de ceux qui pensent, consciemment ou non, l'enseignement (Chevallard, 1991). Afin d'entrer dans la classe, il doit perdre les apprêts de la science et prendre ceux de l'école, être programmable, c'est à dire découpable en différentes unités, et pouvoir s'enchâsser dans l'ensemble de l'histoire scolaire. Par ailleurs tout en restant en contact avec l'histoire scientifique de laquelle il tire sa légitimité, il doit aussi être jugé comme utile par la société en correspondant à ce qu'elle attend comme enseignement fait à ses enfants (Chevallard, 1991).

3 Émanation de l'État, l'histoire scolaire est une histoire institutionnelle qui se trouve contrainte par ce dernier. Les programmes apparaissent alors comme des objets de politique publique, et les commissions ou instances qui sont en charge de leurs compositions sont objets de critiques, qu'elles viennent de l'institution, des enseignants ou des chercheurs. Peuvent s'y heurter les temps courts des ministères au temps long de l'école (Legris, 2010b). Les éléments douloureux du passé y sont passés sous silence, écartés du récit pour différentes raisons touchant à des questions de légitimité du foyer institutionnel ou de paix sociale (Ferro, 1985, 2002), alors que ceux plus glorieux y apparaissent en exergue, et ce à différentes fins, la pacification sociale comme l'amélioration de l'image que la société donne d'elle-même, le tout étant conditionné par leurs évolutions sociales et politiques (Delissen, 2007; Lécureur, 2008). Car c'est bien d'elle-même que parle la société à travers son histoire scolaire, et ce même s'il arrive qu'elle traite d'autres contrées.

4 L'identité, et tout ce qu'elle entraîne de tension, se retrouve donc au centre des problématiques liées à la construction de l'histoire scolaire. L'identité est d'abord celle de l'État et par elle-même celle de la nation, mais elle peut aussi être celle de groupes distincts portant pour eux-mêmes une représentation du passé mémorielle dans la plupart du temps. Face à l'oubli de leur identité, conséquence parfois de la pacification, dans le récit commun, se forgent des contre histoires qui n'ont pour autre but que de trouver leur place et la légitimité dans le récit commun, y parvenant parfois (Ferro, 1985). Cette identité peut aussi toucher des groupes nostalgiques d'une représentation historique plus positive pour la nation, face à une histoire, selon eux, plus encline à montrer les erreurs que les gloires du passé (Chambarlhac, 2012). Pour ce faire, ils peuvent aussi tenter d'acquérir par le pouvoir politique une influence sur ce qui est inscrit, ce qui peut conduire lors de leur arrivée au pouvoir à des lois dites mémorielles (Cajani, 2008). On en revient alors à l'action du politique. Ces groupes offrent d'autres représentations du passé qui, bien que moins diffusées que celle de l'école, la combattent. À travers les commémorations, le cinéma ou encore les journaux, l'histoire est omniprésente dans la société, influençant les élèves, les enseignants et donc le savoir transmis.

$5 \quad$ L'histoire scolaire porte en elle des finalités qui dépassent le simple apprentissage par l'enfant des grandes lignes du passé. Dans un premier temps, l'histoire sert à la formation des bons citoyens, en accord avec les attentes du temps, sujets attachés à la nation à travers son passé, ou citoyens éclairés capables de se placer dans l'histoire, de prendre du recul face aux événements, et de développer un esprit critique (TutiauxGuillon, 2009). Touchant tout autant à l'identité de chacun et la citoyenneté, il peut par ailleurs s'agir de créer un attachement vis-à-vis d'une institution supra-étatique, comme peut l'être l'Union Européenne (Legris, 2010a). Mais à travers l'élève, l'action que l'on conduit vise alors aussi la société. Le bon citoyen apprend aussi le vivre 
ensemble et c'est autour de ces idées, et d'un passé commun que se construit la cohésion nationale, l'identité commune. Dans tous les cas, les éléments montrés, la façon de les présenter, pourront être différents. Et face à une histoire scientifique en grande partie détachée des impératifs institutionnels, des écarts pourront se creuser, des différences entre les récits soumettant par ailleurs cette histoire scolaire à la critique des scientifiques. La construction de l'histoire scolaire est donc une question socialement vive, tant dans la société que dans la discipline et sa didactique, tout en sachant que la didactique de l'histoire est encore une discipline qui réclame de la recherche une émancipation des cadres théoriques produits par les didactiques des disciplines scientifiques.

Ce numéro de Questions Vives a fait l'objet d'un appel à contributions totalement ouvert, sans qu'aucun auteur n'ait été pressenti au départ. Il nous semble que les auteurs qui ont répondu à l'appel illustrent de façon originale l'une ou l'autre des facettes de nos questions à la didactique de l'histoire. Nous laissons au lecteur curieux le soin de vérifier de quelle manière. Que chacun soit ici remercié pour sa contribution.

\section{BIBLIOGRAPHIE}

Audigier, F. (1988). Savoir enseignés - savoir savant. Autour de la problématique du colloque. Dans Actes des rencontres sur la didactique de l'histoire, de la géographie, des sciences économiques et sociales. Troisième rencontre nationale sur la didactique de l'histoire, de la géographie, des sciences économiques et sociales. Savoirs enseignés/savoirs savants, (pp. 55-69). Paris : INRP. Cajani, L. (2008). L'histoire, les lois, les mémoires. Sur quelques conflits récents en Europe. Revue française de pédagogie, 165(4), 65-76.

Cariou, D. (2012). Historisation de la didactique de l'histoire : démarches de pensée historienne et apprentissage de l'histoire. Dans M.-L. Elalouf, A. Robert, A. Belhadjin \& M.-F. Bishop (Éds), Les didactiques en question(s). État des lieux et perspectives pour la recherche et la formation (pp. 69-78). Bruxelles : De Boeck.

Chambarlhac, V. (2012). Les prémisses d'une restauration ? L'histoire enseignée saisie par le politique. Histoire@politique. Politique, culture, société, 16. Consulté à l'adresse http:// www.histoire-politique.fr/index.php?numero=16\&rub=pistes\&item=22

Chevallard, Y. (1991). La transposition didactique, du savoir savant au savoir enseigné. Grenoble : La pensée sauvage.

Delissen, A. (2007). La nouvelle bataille des Falaises rouges ? Vingtième Siècle. Revue d'histoire, 94(2), 57-71. doi : 10.3917/ving.094.0057

Ferro, M. (1985). L'histoire sous surveillance. Paris : Calmann-Levy.

Ferro, M. (2002). Les tabous de l'Histoire. Paris : Nil.

Ginzburg, C. (2001). A distance. Neuf essais sur le point de vue en histoire. Paris : Gallimard. 
Heimberg, C. (2007, septembre). En quoi la compétence d'exercer un regard critique sur le monde et ses questions vives est-elle reliée à l'histoire et à ses questions fondamentales. Communication présentée lors du colloque : Compétences et socialisations, Montpellier. Consulté à l'adresse http:// www.unige.ch/fapse/edhice/textesenligne/textesedhice/heimberg.2007.pdf

Lécureur, B. (2008). L'autre Allemagne, la résistance intérieure au nazisme, un aspect particulier des manuels d'Histoire allemands publiés depuis 1950. Tréma, 29, 33-47. doi : 10.4000/trema.730

Legris, P. (2010a). L'écriture des programmes d'histoire en France (1944 - 2010). Sociologie historique d'un instrument d'une politique éducative. Mémoire de doctorat en sciences politique non publié. Paris-I Panthéon-Sorbonne, Paris.

Legris, P. (2010b). Les programmes d'histoire en France : la construction progressive d'une “citoyenneté plurielle" (1980-2010). Histoire de l'éducation, 126, 121-154.

Moniot, H. (1993). Didactique de l'Histoire. Paris : Nathan.

Tutiaux-Guillon, N. (2009). L'histoire scolaire française entre deux modèles : contenus, pratiques et finalités. Revue française d'éducation comparée, 4, 105-116.

\section{AUTEUR}

\section{CHRISTIAN ROUX}

Aix-Marseille Université 